\title{
INVESTIGACIÓN/RESEARCH
}

Recibido: 28/10/2013-----Aceptado: 10/01/2013-----Publicado: 15/03/2014

\section{PROPUESTA DIDÁCTICA BASADA EN LAS TÉCNICAS DE GIANNI RODARI Y LOS JUEGOS DE EDWARD DE BONO PARA LA PRODUCCIÓN DE TEXTOS LITERARIOS CREATIVOS}

Maritza Álvarez Rodríguez¹: Universidad Pedagógica Experimental Libertador de Caracas. Venezuela.

Isamary2007@yahoo.es

\section{RESUMEN}

La creación de textos literarios representa una expresión del pensamiento creativo, en consideración a la generación de ideas nuevas y diferentes a lo elaborado frecuentemente en las aulas. El estudio se planteó construir una propuesta didáctica para la producción de textos creativos y literarios basados en las Técnicas de Gianni Rodari y los juegos didácticos de Edward de Bono. La investigación se enmarcó en el paradigma sociocrítico con un enfoque etnosociológico y fenomenológico social. Metodológicamente se recogió información en el aula proveniente de estudiantes universitarios de la especialidad de Educación Integral en el Instituto Pedagógico de Miranda José Manuel Siso Martínez.

PALABRAS CLAVE: Texto creativo - Producción escrita - Juegos didácticos.

\footnotetext{
${ }^{1}$ Maritza Álvarez Rodríguez: Universidad Pedagógica Experimental Libertador. Instituto Pedagógico de Miranda José Manuel Siso Martínez.

Correo: Isamary2007@yahoo.es
} 


\title{
PROPOSAL BASED LEARNING TECHNIQUES GIANNI RODARI AND GAMES EDWARD DE BONO FOR CREATIVE PRODUCTION OF LITERARY TEXTS
}

\begin{abstract}
The creation of literary texts is an expression of creative thinking, considering the generation of new ideas and different to the elaborate frequently in classrooms. The study was proposed to build a methodological approach to producing creative texts and literary techniques based on Gianni Rodari and educational games of Edward de Bono. The research was part of the paradigm sociocritic a phenomenological approach and social etnosociológico. Methodologically information was collected from classroom college students specializing in Global Education at the Pedagogical Institute of José Manuel Siso Martínez Miranda. Keywords: Children's Literature, creative text, writing, learning games.
\end{abstract}

KEYWORDS: Creative text - Written production - Educational games.

\section{INTRODUCCIÓN}

La Literatura es un vehículo de acceso único al poder de la lengua, a poder de apropiación de la realidad que las habilidades lingüísticas confieren, como decía Heidegger "es apropiarse del más peligroso de los bienes" ${ }^{2}$. En este sentido se analizan los aspectos más importantes de lo que se denomina "esritura creativa" relacionadas con las técnicas presentadas por Gianni Rodari en su libro "Gramática de la Fantasía". Se entiende por escritura creativa a todo proceso que genere ideas novedosas orientadas al desarrollo del conocimiento a través de la actividad escritural en combinación con los juegos didácticos creativos propuestos por De Bono $(2007)^{3}$.

\section{OBJETIVOS}

\subsection{Objetivo General}

Generar una propuesta didáctica basada en las técnicas de Gianni Rodari y los juegos creativos propuestos por De Bono para la metodología de la producción de textos escritos en la literatura infantil en estudiantes de Educación Integral del IPMJMSM.

\footnotetext{
2 Heidegger (1987) Página 37.

3 De Bono (2007). Juegos Didácticos.
} 


\subsection{Objetivos Específicos}

- Identificar el proceso de enseñanza y aprendizaje utilizados por los docentes y estudiantes del IPMJMSM para la producción de textos literarios creativos.

- Identificar los elementos creativos en la producción de textos literarios producidos por los estudiantes de Educación Integral la producción de textos literarios y su relación con la creatividad en el ámbito de la formación docente.

- Diseñar un cuerpo de estrategias creativas para enseñar a los estudiantes de Educación Integral a elaborar textos literarios creativos según algunas técnicas para la producción escrita de Gianni Rodari y juegos creativos propuestos por De Bono.

- Describir la relación que existe entre textos creativos y literatura infantil, en atención a docentes y estudiantes de Educación Integral.

- Establecer un conjunto de estrategias creativas que puedan servir de base para la generación de una propuesta didáctica basada en las técnicas de Gianni Rodari y los juegos creativos de De Bono para la metodología de textos creativos en literatura infantil. académicos.

\section{METODOLOGÍA}

La investigación se enmarcó en el paradigma sociocrítico con un enfoque etnosociológico y fenomenológico social. Metodológicamente se recogió información en el aula proveniente de estudiantes universitarios de la especialidad de Educación Integral en el Instituto Pedagógico de Miranda José Manuel Siso Martínez. El estudio consta de cuatro fases: (1) Reflexiones a partir de revisiones teóricas y bibliográficas sobre el texto creativo y su producción; (2) Categorización de la información suministrada por los actores (Estudiantes de E.I.); (3) Integración de las categorías y triangulación de las fuentes para establecer conexiones entre hallazgos y teorías; (4) Aportes de la propuesta didáctica. Los resultados se orientaron a demostrar que la fusión efectiva de las Técnicas de Rodari y los Juegos Didácticos e Edward de Bono cambian la imagen que tienen los estudiantes sobre el proceso de escritura. La estrategia utilizada fue el taller donde se explicaron las cuarenta y cinco (45) técnicas y los juegos didácticos porque se consideró la preparación previa para redactar el texto creativo que debía pertenecer a un género literario (cuentos, novela, fábula, leyenda, etc.). Posteriormente el texto se corrige y puede ser mejorado por el estudiante. De esta forma se evidencia que la escritura puede ser un proceso dinámico, interesante, instructivo y creativo.

\section{RESULTADOS}

En relación con el término escritura "creativa", hay algunas objeciones. Algunos autores consideran que toda escritura es creativa y, por tanto prefieren hablar de escritura de ficción o de talleres de creación literaria, otros opinan que no siempre la escritura es creativa. La autora de este estudio considera que la propuesta de escritura creativa se fundamenta en un marco pluridisciplinario que incluye 
disciplinas como pedagogía, literatura, lingüística, psicología, semiótica, entre otros, como las teorías de la creatividad, el aprendizaje significativo, la estética de la recepción y la pedagogía de la creatividad y el juego.

El déficit en la producción de textos en los estudiantes universitarios es un abismo inhóspito porque no se les da la oportunidad de escribir en libertad por las exigencias de algunos cursos donde solo se evalúan los aspectos formales de la escritura, destrezas que no se desarrollan ni en la escuela, ni en los liceos y mucho menos en las universidades venezolanas, donde no se propician espacios para la escritura creativa, es aquí donde la investigación cobra relevancia puesto que propone la producción de textos creativos en literatura infantil y este trabajo aspira cubrir las necesidades de formación literaria en los futuros maestros de la patria.

\section{DISCUSIÓN}

Entre los hallazgos más importantes se encuentran: la fusión efectiva de las Técnicas de Rodari y el descubrimiento emergente de una herramienta titulada la aventura literaria, además de un nuevo concepto de la redacción.

\subsection{La Aventura Literaria}

Es una herramienta novedosa que permite abordar el mundo de la literatura desde una perspectiva integradora tanto de los conceptos fundamentales de la materia como de sus posibilidades expresivas en un formato que cuenta por sí mismo con un gran poder de atracción sobre los usuarios y usuarias, responde a una situación habitual entre el estudiante que cursa la Educación Universitaria. Este colectivo se enfrenta a la literatura como quien es abandonado en medio de un frondoso bosque sin mapa ni brújula ni guía de ningún tipo. Algunos salen indemnes gracias a su intuición o a su buena suerte, otros se pierden en una febril actividad que nos les conduce a ninguna parte, pero otros muchos se dejan abatir por la falta de perspectiva global, la maraña terminológica, la espesura de los conceptos o la dificultad de alcanzar los objetivos propuestos. Para unos y para otros debe haber una formación común y básica que les permita orientarse, procurarse recursos y salir airosos de la situación en más o menos tiempo, en función de las capacidades de cada uno; a la vez, será necesario disponer de rutas alternativas de aprendizaje para quienes deseen disfrutar del trayecto y hasta para aquellos que, dotados de un espíritu más emprendedor, se atrevan una exploración personal para dar con una solución creativa y original al reto planteado.

Los autores, sin ocultar la dificultad de la tarea que supone dominar los conceptos básicos de la literatura, pretenden ofrecer un banco de propuestas que haga compatible el deseo de explorar lo desconocido con la obligación de formarse. Es decir, la aventura literaria se integra en un proyecto más amplio: aprender a aprender en el seno de una sociedad compleja y cambiante que pone al alcance de sus miembros herramientas cada vez más sofisticadas. Dominar esas herramientas, los medios informáticos, es un modo para facilitar la formación integral del 
aprendiz y su integración en la comunidad cultural, de hechos y de valores, que la lengua de esa comunidad vehicula.

La aventura literaria no pretende dar más de lo mismo, sino organizar la materia de una manera más atractiva, aprovechar la motivación añadida que las nuevas tecnologías ofrecen para facilitar la integración social del aprendiz y desarrollar su creatividad.

\subsection{Fundamentación Didáctica}

La aventura literaria se sustenta en un soporte triple: una concepción de la lengua, una concepción de la literatura y una concepción de la actividad didáctica.

\subsubsection{Concepción de la Lengua}

La concepción que sobre la lengua se tenga no es la única variable que ha de intervenir a la hora de decidir acerca de la formulación y organización de los objetivos y los contenidos de un currículo de lengua ni sobre el enfoque metodológico que se ha de dar a la enseñanza de esta materia o a su evaluación y, sin embargo, es el criterio esencial entre todos los otros por ser el que otorga a aquéllos su especificidad.

Se define la lengua como un proceso de producción y de negociación de significados entre interlocutores que pueden estar mediatizados por situaciones culturales diferentes. Estas pinceladas caracterizan y amplían la concepción:

La lengua es un instrumento de acceso a una cultura, porque la lengua es una realidad en perpetuo movimiento, que no existe ni significa fuera de cada una de las interacciones humanas en las que es utilizada.

El objetivo de las actividades de enseñanza-aprendizaje de una lengua será aprender a interaccionar adecuadamente en diversos contextos, es decir, la adquisición de una competencia comunicativa.

La apropiación de una lengua, o mejorar en su conocimiento, es un instrumento imprescindible para adquirir o ampliar una competencia cultural.

La lengua favorece los procesos de maduración: al comienzo de la etapa universitaria una buena parte de la experiencia histórica acumulada por la comunidad de hablantes le ha sido ya transmitida al adolescente, a través de las diversas interacciones mediadas por el lenguaje en las que ha transcurrido la infancia de éste. En esa dinámica de transmisión, que no es idéntica para todos ni en su desarrollo ni en sus resultados, pues varía según los códigos lingüísticos y las prácticas sociales presentes en aquellas interacciones, se ha ido configurando el uso de la lengua, el sistema de valores, creencias y hábitos a la vez que la estructura y características específicas de la conciencia de cada cual. 
Las actividades de comprensión y producción de textos de carácter literario, con el análisis y la utilización de los distintos recursos y técnicas literarias, son la expresión máxima del uso autorreflexivo del lenguaje. Son también una ocasión privilegiada para potenciar el desarrollo del pensamiento abstracto hipotéticodeductivo.

\subsubsection{Concepción de la Literatura}

La lengua es simultáneamente depósito de la cultura de la comunidad que la emplea e instrumento de acceso a esa cultura. La complementariedad de ambos aspectos se manifiesta de modo muy especial en la literatura. En ella, la lengua desborda el papel de instrumento de producción y negociación de la significación para llamar la atención sobre el funcionamiento de los mismos discursos, pues éstos se presentan organizados de un modo peculiar que obliga a reflexionar sobre la estructura del propio mensaje. Por ello, ciertas posibilidades de la actualización del lenguaje aparecen mucho más marcadas en la literatura, ya sea oral o escrita. $Y$ los textos procedentes de ambas tradiciones codifican de una forma tan particular la experiencia, la mentalidad y la forma de entender el mundo colectivas de un pueblo que reclaman un oyente o lector activo al que proporcionan diversión y placer a la vez que le transmiten patrones culturales, modos de interpretar la realidad, suministrándole elementos para reflexionar sobre sí mismo y sobre el mundo y para fundamentar sus juicios y opiniones. Por ello, la literatura se constituye en uno de los canales de participación cultural del ciudadano, lo mismo en su calidad de receptor que de eventual creador. Así, la literatura, en su especificidad, trasciende mucho más que otro tipo de interacciones comunicativas, el enriquecimiento de los usos verbales hasta afectar al mundo de los valores personales y de las opciones ideológicas.

\subsubsection{La Concepción de la Actividad Didáctica}

Un sistema de enseñanza sólo podrá facilitar la construcción de la propia identidad del aprendiz si es capaz de orientarle entre los estímulos diversos, complejos y, con frecuencia, contradictorios a los que está sometido y de proporcionarle elementos para el análisis y para la comprensión y apropiación significativa de aquéllos. Se tratará, en la práctica, de la puesta en pie de un modelo de interacción educativa que ayude a los estudiantes a construir autónomamente una competencia intercultural, integradora de las prácticas más modernas con las tradiciones culturales en las que se desarrolla la personalidad individual.

Por ello, las prácticas de aula habrán de ser tales que proporcionen al aprendiz las ayudas necesarias para tomar conciencia de los implícitos que determinan su propio capital cultural, para desentrañar los que configuran los capitales ajenos y para asegurar el diálogo entre ellos, creando así las condiciones para la construcción intercultural de la casa de la conciencia. A través de dichas prácticas de aula se tratará de: (a) Instaurar una pedagogía orientada a la producción y negociación de significados culturales haciendo especial hincapié en los numerosos implícitos presentes en las interacciones lingüísticas y literarias; (b) Privilegiar en el 
aula la presentación, análisis y reconstrucción de aquellas situaciones de comunicación culturalmente más ricas y con los medios técnicos más actuales y, (c) Potenciar para ello aquellas metodologías, innovadoras o tradicionales, que permitan un uso de la lengua y un diálogo cultural auténticos.

\subsection{Criterios}

A continuación se describen los criterios, de entre los propuestos por el curso, más adecuados para alcanzar los objetivos planteados a través del empleo de la aventura literaria:

Producir textos escritos de diferentes tipos (narrativos, descriptivos), adecuándolos a la situación de comunicación, utilizando la estructura organizativa de cada uno y respetando los criterios de corrección.

Este criterio trata de comprobar que los estudiantes son capaces de producir textos narrativos, descriptivos, expositivos y argumentativos adecuados a la situación comunicativa, organizando sus partes y componentes de acuerdo con las estructuras propias de dichos tipos de texto. A su vez, se debe mostrar un uso apropiado de los procedimientos de cohesión (usos anafóricos de los pronombres, repeticiones, sustituciones, elipsis, entre otros), de los elementos de conexión (conjunciones, adverbios, locuciones adverbiales, entre otros) y de construcciones sintácticas y compuestas.

Planificar y llevar a cabo, individualmente o en equipo, la consulta de diversas fuentes de información mediante el manejo de índices, fichas y otros sistemas de clasificación de fuentes, en el marco de trabajos sencillos de investigación.

El criterio centra su atención en la capacidad del estudiante para efectuar investigaciones asequibles a su edad y formación, tanto de forma individual como en equipo. Exige planificar la actividad, organizar la información necesaria, contrastarla y deducir conclusiones objetivas que sean útiles para el fin que se pretende. El estudiante deberá conocer y manejar los sistemas de clasificación (ficheros, índices, entre otros) de la biblioteca del centro u otras semejantes que sean de posible consulta.

Utilizar las propias ideas y experiencias para la producción de textos de intención literaria, empleando conscientemente estructuras de género y procedimientos retóricos y recurriendo a modelos de la tradición literaria.

El criterio pretende demostrar si los estudiantes son capaces de iniciar un proceso de creación de un texto literario sirviéndose de sus propias vivencias y emociones, así como de aquellos ejemplos de autores consagrados que la literatura les ofrece. Mostrarán una cierta desenvoltura en el ejemplo de las estructuras propias de cada género y usarán los recursos expresivos del lenguaje literario, tanto en prosa como en verso, que se haya trabajando en la práctica pedagógica del aula. 
Establecer relaciones entre obras, autores y movimientos que constituyen una referente clave en la historia de la Literatura y los elementos más destacados del contexto cultural, social e histórico en que aparecen.

El criterio de comprobar en el alumno su compresión del fenómeno literario como una actividad comunicativa estética y, a su vez, como un producto social y cultural situado en un contexto histórico determinado. Para ello es necesario que el estudiante conozca los autores, obras y hechos literarios más representativos de la historia de la Literatura y los relacione con los acontecimientos culturales y sociales existentes en el contexto de su producción.

\subsection{Instrumentos}

Serán instrumentos preferentes de evaluación los archivos creados por el aprendiz y en los cuales el docente tiene acceso no sólo al producto final, sino a los sucesivos borradores intermedios utilizados y al listado de archivos abiertos de modo que se evalúa también el proceso.

\subsection{Redacción}

El código escrito no se limita a plasmar mediante signos lo que hablamos sino que constituye un código completo e independiente del código oral. Cuando nos expresamos por escrito debemos tener en cuenta la adecuación, la estructura del texto, la organización jerárquica de las ideas, la corrección gramatical, además de la ortografía y la caligrafía. Todo esto supone una gran cantidad de conocimientos que, según Frank Smith y Stephen Krashen, no pueden ser aprendidos conscientemente con ejercicios de gramática porque la lengua es excesivamente compleja, sino de manera inconsciente mediante la lectura. Para aprender a escribir hay que leer como un emisor (escritor), y de este modo aprender a usar el lenguaje escrito de la misma manera que lo usan los buenos escritores.Un texto bien escrito tiene que tener unas características específicas de organización que se engloban bajo los términos de coherencia y cohesión. La coherencia es una propiedad de los textos bien formados que permite que sean vistos como un todo unitario, de manera que las ideas secundarias aporten la información precisa relacionada con el tema central. Un escrito es coherente cuando su tema es claro y está desarrollado ordenadamente con progresión en la información y sin enunciados contradictorios; mientras que la coherencia es una cuestión semántica, la cohesión es una cuestión lingüística y gramatical puesto que trata de la relación sintáctica entre las partes del discurso para que este sea coherente.

\subsection{Evidencias sobre la producción de textos escritos creativos en la Universidad}

De acuerdo con lo expuesto anteriormente el problema que la investigadora se planteó guarda relación con los textos escritos creativos. En respuesta a la problemática, se propone el desarrollo de la escritura creativa en estudiantes universitarios del Instituto Pedagógico de Miranda José Manuel Siso Martínez 
(IPMJMSM) en la especialidad de Educación Integral (EI). Desde el empirismo de la investigadora al administrar el curso literatura infantil y a partir del establecimiento de redes comunicacionales con los colegas del instituto e incluso con los docentes contratados que administran el curso, se ha podido evidenciar que los estudiantes universitarios de la especialidad de EI desconocen el poder de la escritura creativa porque por lo general escriben por obligación sin dejar que intervenga la imaginación y la fantasía debido a que el texto que producen es académico (ensayo, redacción, entre otros) y la propuesta de esta investigación se refiere a textos literarios (cuento, mito, leyenda, fábula, poema, entre otros). El programa del curso Literatura Infantil profundiza en la elaboración de textos literarios, en la lectura y el análisis de autores relevantes dentro de la literatura infantil. Es importante destacar que estas mismas redes comunicacionales y la experiencia docente demuestra que la falla en la producción escrita es un problema inherente a los estudiantes universitarios, por tal motivo es una situación que afecta a todas las especialidades que administra el IPMJMSM. Como evidencia de lo anterior se tiene la referencia de los docentes del área de Lengua y Literatura donde se evidencia la concepción que tienen los estudiantes sobre la escritura, la redacción y la producción de textos libres. En palabras de una profesora contratada del IPMJMSM... "Los estudiantes no tienen habilidades para la escritura, no pueden desarrollar una idea, solo copian si es un texto elaborado en el aula...pero si es un trabajo escrito con recortar y pegar de internet el escrito es más fácil". Para la autora de la tesis escribir es un proceso creativo, donde la imaginación debe jugar un papel importante y es justamente el docente quien debe motivar a escribir pero dada la libertad... justamente como lo dice Rodari (1980) " ${ }^{4}$ "No para que todos seamos escritores sino para que seamos libres". A continuación un gráfico donde se explica la relación entre escribir, escritura y redacción.
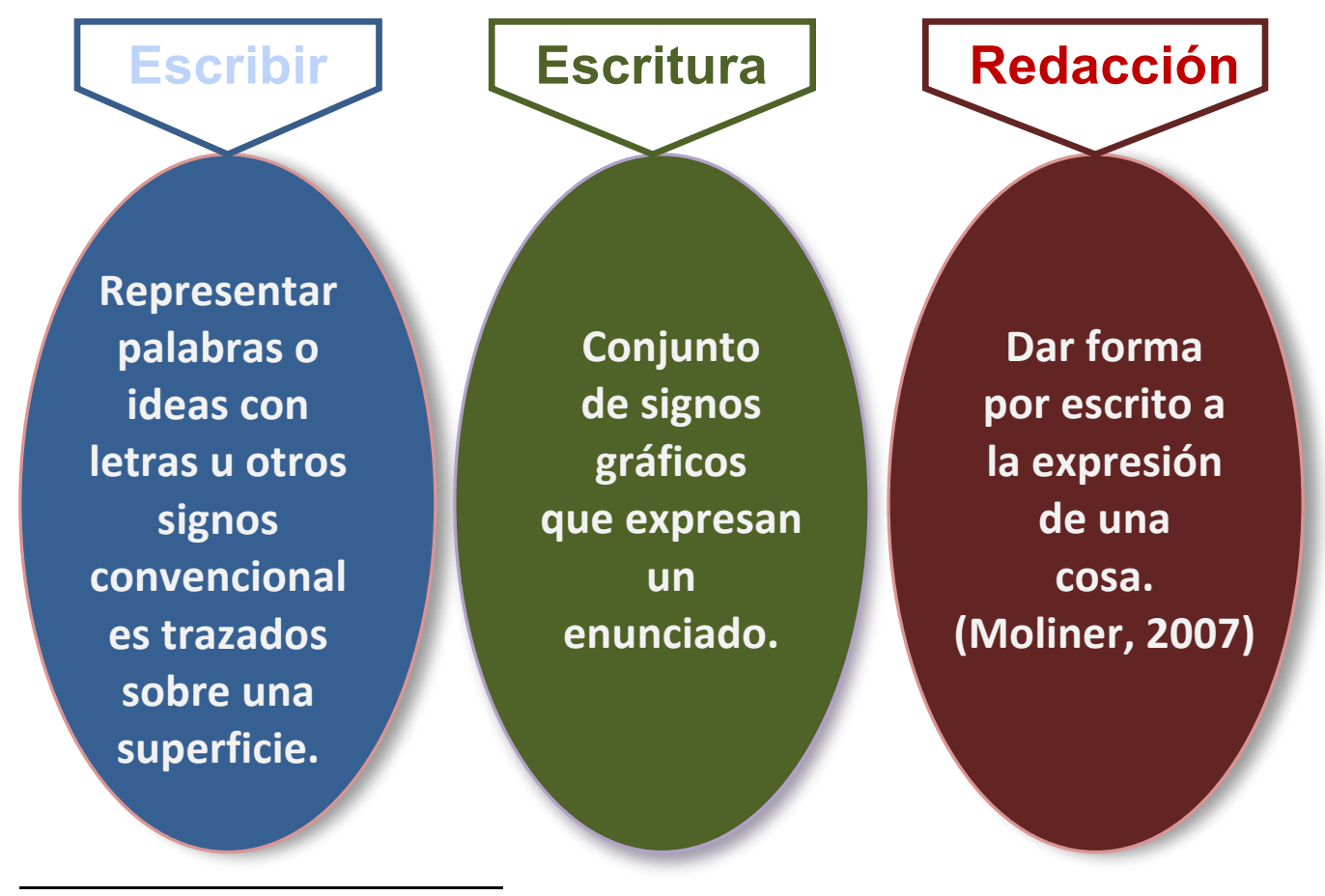


\section{Gráfico 1. Conceptualización de Escribir, Escritura y Redacción ${ }^{5}$.}

Las ideas anteriores justifican la necesidad de generar un mecanismo que permita la creación de textos creativos como vía potenciadora del lenguaje y colaboradora del proceso educativo. Es importante destacar que los estudiantes de E.I. que sirven de base a esta investigación, ejercerán la profesión docente, o se encuentran desempeñando ese rol, con una población que tiene características bien particulares, estos son los niños y las niñas que cursan de primero a sexto grado, o bien una población cuyo pensamiento se encuentra en constante desarrollo. En palabras de Montessori (2003) son una esponja y tal como se les potencie su inteligencia y pensamiento en esta medida serán sus producciones.

Son muchas las preguntas que un investigador $y / 0$ un docente se plantean frente a la problemática de la enseñanza de la producción escrita y su respectiva evaluación. A modo de ejemplo, cabe preguntarse cuando se evalúan los trabajos de los estudiantes: ¿qué aspectos se toman en consideración?, ¿cómo es posible obtener información sobre el nivel de desarrollo de un escritor? ¿cómo se pueden identificar los aspectos formales de la escritura?, ¿se examina sólo el producto escrito o se intenta indagar en los procesos y estrategias que generaron ese producto?

Al respecto, Cassany ${ }^{5}$ (1996), opina que la distinción entre corrección y evaluación del discurso escrito puede ser aclaradora. Según este autor, la primera interesa más a maestros o educadores preocupados por la revisión de los trabajos de sus estudiantes como parte del desarrollo de la habilidad escrita. Su objetivo central es ayudarlos a enmendar sus errores y avanzar en su manejo de las estructuras y recursos necesarios para llegar a elaborar textos coherentes y cohesionados en diversas tipologías. Este autor considera que la evaluación es preocupación de quienes deben diseñar pruebas o instrumentos que pretendan alcanzar índices apropiados de validez y confiabilidad, muchas veces, con el objetivo de determinar el nivel de competencia discursiva de un grupo de sujetos.

La obra que da origen a esta investigación es la Gramática de la Fantasía del italiano Gianni Rodari ${ }^{6}$ (1979), donde se proponen una serie de técnicas para la producción de textos creativos y fantásticos. Es importante destacar que la investigadora adaptó las técnicas del autor mencionado para lograr que los estudiantes produzcan a través de la metacognición textos libres, creativos, fantásticos, originales adaptados a los géneros literarios (cuento, poema, fábula, leyenda, mitos, entre otros).

\footnotetext{
${ }^{5}$ Álvarez Maritza (2012). Tesis Doctoral.

${ }^{5}$ Cassany (1996) Describir el escribir.

${ }^{6}$ Rodari (1979). Gramática de la Fantasía
} 
En su experiencia Rodari, anotaba "no las historias que contaba, sino como nacían, los trucos que iba descubriendo o creía descubrir para poner en movimiento las palabras y las imágenes" (p.14). Esto es importante dado que demuestra la posibilidad del ser humano de desarrollar su potencial creativo dándole cabida a la intervención de la imaginación y la fantasía. Hacia 1948, el autor señala que empezó a escribir para los niños lo cual dio origen a las diversas ideas en torno a la gramática de la fantasía. "En el marco de esta ficción no muy original exponía, medio en serio, medio en broma, algunas técnicas sencillas de invención: las mismas que he divulgado luego en todas las escuelas en las que he ido a contar historias y a responder las preguntas de los niños. Porque siempre hay un niño que pregunta: ¿Cómo se hace para inventar historias? y merece una respuesta honesta". (p.15). ${ }^{7}$

Paralelo a la aplicación de las técnicas de Rodari se utilizaron los ejercicios para desarrollar la mente propuestos por De Bono (2007). De esta manera, la investigadora intentó vincular la fantasía propuesta por Rodari con la creatividad ensayada por De Bono. Se sugirieron las características del docente mediador a fin de que éste ayude a los estudiantes a usar y transferir la creatividad al área de literatura y a la vida diaria. Con esta relación fantástica-creativa (negrita de la autora) se favorecer los tres grandes componentes del ingenio: codificación, combinación y comparación selectiva.

Finalmente, la investigadora, buscó desarrollar la creatividad en la producción de textos en estudiantes universitarios de la Especialidad de Educación Integral.

\section{REFERENCIAS}

Almacellas y Otros (2000). Educar la inteligencia. Argentina: Galeón.

Álvarez, G. (1996) Textos y discursos. Introducción a la lingüística del texto, Concepción: Universidad de Concepción.

Applebee, A.; Langer, J.; Mullis, I.; Lathmam, A. y Gentille, C. (1994) Writing report card. Washington, DC: Office of Educational Research and Improvement, US Department of Education. NAEP 1992:

Azcárate, P.; Cuesta, J. y Navarrete, A. (1994). Presupuestos iniciales para un trabajo de investigación sobre formación del profesorado. Revista Investigación en la Escuela.

Azpúrua, F. (2004). El interaccionismo simbólico y la fenomenología social. Sus principios y contribuciones en la etnosociología. En arbitraje. Vol. 2, № 3.

\footnotetext{
${ }^{7}$ Rodari (1979) Gramática de la Fantasía
} 
Bellemin-Nöel. (1983). Notas sobre la Fantasía. México: The colors of space.

Calsamiglia, H (1993). Singularidades de la elaboración textual: aspectos de la enunciación escrita. El enfoque comunicativo de la enseñanza de la lengua. Barcelona, Paidós Papeles de Pedagogía/14.

Cassany, D. (1999). Describir el escribir. Barcelona: Paidós.

Cervera, J. (1985). La literatura infantil en la educación básica. Madrid: Cincel. Consejo Nacional de Universidades. (1985).

Cooper y Odell. (1977). Evaluating writing: describing, measuring, judging, USA: NCTE.

De Bono, E. (2007). Creatividad. España: Mcquady Group Inc.

Del Villar, F. (1994). El Diario de los profesores de educación. Un instrumento de investigación y formación docente. Revista Española de Educación Física y Deportes, 4.

Desrosiers. (1971). Una introducción a la teoría literaria. México. F.C.E.

Drac (2010). Barcelona, España: Espalsa-Calpe, C.A.

Foucault, M. (1996). De lenguaje y literatura. Barcelona: Paidós.

Frías, M. (1996). Procesos creativos para la construcción de textos. Bogotá: Magisterio.

Geertz, C. (1991). La interpretación de las culturas. Barcelona: Gedisa.

Glaser, B. y Strauss, A. (1967). El desarrollo de la teoría fundada. Chicago, Illinois: Aldine.

Goetz, J. y La Compte, M. (1988). Etnografía y diseño cualitativo en investigación educativa. Madrid: Murap, S.A.

Guilford, J. y Strom, R. (1978). Creatividad y educación. Buenos Aires: Paidós.

Hairston, M. (1982). Winds of change: Thomas Kuhn and the revolution in the teaching of writing. College Composition and Communication.

Hallman, R. (1975). Condiciones necesarias y suficientes de la creatividad. En Curtis y otros. 
Hammer y Strinson. (1994). Didáctica de la lengua y la literatura. España: Universidad de la Mancha.

Heidegger. (1987). Holderling y la esencia de la poesía. España: Icaria.

Held. (1981). Literatura infantil y juvenil. España: Universidad de Castilla La Mancha.

IRA y NCTE. (1994). Standards for the assessment of reading and writing, IRA y NCTE: USA.

Jakobson, R. (1975). Lingüística y poética. Trad.esp. En Ensayos de lingüística general. Barcelona: Seix Barral.

Lacau, M. (1966). Gramática. Madrid: Grijalbo.

Lapassade, G. (1991). L'ethnosociologie. Paris: Meridiens Rdincrsiedk.

Lavelle, E. (1993). Development and validation of an inventory to assess processes in college composition. British Journal of Educational Psychology.

Lofland, J. (1971). Analyzing social selting: a guide to cualitative observation and analisys. Belmont cal. Wadsworth.

Logan, L. y Logan, V. (1980). Estrategias para la enseñanza creativa. Barcelona: Oikos - Tau.

Martínez, M. (2004). Ciencia y arte en la metodología cualitativa. México: Trillas.

Makinkovich. (2002). Producir texto. Chile: Ediciones Universitarias.

Maxwell, A. (1996). Qualitative Reseach Design. An interactive Approach. California: Sage Publications.

Mednick, S. (1964). The associative basis of the creative process. Psychological Review.

Moliner. (2007). Diccionario. Argentina: Paidós.

Montessori. (2003). La casa del saber. Caracas: Santillana.

Myers, M. (1980). A procedure for writing assessment and holistic scoring, Urbana, Illinois: NCTE.

North, S. (1987). The making of knowledge in composition. London and Portsmouth, NH: Heinemann. 
Pandit, N. (1996). La creación la teoría: Una aplicación reciente del método de teoría fundamental. En: Informe cualitativo, № 4, Dic 1996. Vol. 2.

Parodi, G. (2000). Producción de texto. Buenos Aires: Eudeva.

Pérez, G. (1998). Investigación cualitativa: Retos e interrogantes. Métodos (Vol. 1). Madrid: La Muralla, S.A.

Pérez, G. (1994). Investigación cualitativa: Retos e interrogantes. Madrid: La Muralla, S.A.

Pérez, G. (1998b). Investigación cualitativa. Retos e interrogantes II. Técnicas y análisis e datos. Madrid: La Muralla, S.A.

Picón, G. (2006). Una teoría y un método para investigar y promover el cambio en educación. Trabajo no Publicado. Instituto Pedagógico de Caracas UNESR-UPEL, Caracas.

Porlán, R. (1987). El maestro como investigador en el aula. Investigar para conocer, conocer para enseñar. Revista Investigación en la Escuela, 1.

Porlán, R. y Martín, J. (1991). El diario del profesor. Sevilla: Diada.

Rodari, G. (1999). Gramática de la fantasía. Introducción al arte de inventar historias. Bogotá: Panamericana Editorial.

Rodari, G. (1979). Gramática de la fantasía. Barcelona: Ferrán, Pelisa.

Rogers, C. (1975). Hacia una teoría de la creatividad. En El proceso de convertirse en persona. Paidós. Barcelona.

Ruth, L. y Murphy, S. (1988). Designing writing tasks for the assessment of writing, Norwood, NJ: Ablex.

Sánchez, M. de (1998). Desarrollo de las habilidades del pensamiento. México: Trillas.

Sandín, M. (2003). Investigación cualitativa en educación, fundamentos y tradiciones. Madrid: McGraw-Hill/Interamericana de España.

Sandoval. (1997). La literatura infantil. Madrid: La Muralla S.A.

Schutz, A. (1987). Lechecheur et le quotidiem. París. Mérbliens: Rlincrsiecr.

Sendak. (1978). Le Checheur et le quotidien. Paris. Meridiem Relinchisieck.

Sequeida, J. y Seymour, G. (1994). Demandas cognitivas del desarrollo de la expresión escrita en adolescentes. Pensamiento Educativo. 


\section{Maritza Álvarez Rodríguez}

Profesora de Castellano egresada del IPC en 1985 y Magíster en Literatura Hispanoamericana con tesis Mención Publicación en 1993.Doctora en educación 2012. Actualmente forma parte del personal ordinario del Pedagógico de Miranda J.M. Siso Martínez, en la categoría de agregado, donde coordina el área de Literatura Infantil. Publicó varios artículos en las revistas Cohensremi, Sapiens y en el Suplemento Cultural de Últimas Noticias. Fue miembro del Comité editorial de la revista Sapiens y correctora de estilo del Boletín de Educación Alimentaria Pira. Además, ha sido ponente en diversos eventos nacionales e internacionales relacionados con Literatura e Historia. PEI categoría investigador. 